\title{
Basic Statistics of Jevons and Carli Indices under the GBM Price Model
}

\author{
Jacek Biatek ${ }^{1}$
}

\begin{abstract}
Most countries use either the Jevons or Carli index for the calculation of their Consumer Price Index (CPI) at the lowest (elementary) level of aggregation. The choice of the elementary formula for inflation measurement does matter and the effect of the change of the index formula was estimated by the Bureau of Labor Statistics (2001). It has been shown in the literature that the difference between the Carli index and the Jevons index is bounded from below by the variance of the price relatives. In this article, we extend this result, comparing expected values and variances of these sample indices under the assumption that prices are described by a geometric Brownian motion (GBM). We provide formulas for their biases, variances and mean-squared errors.
\end{abstract}

Key words: Consumer price index; geometric Brownian motion; Jevons index; Carli index.

\section{Introduction}

Elementary price indices are used in inflation measurement at the lowest level of aggregation. The choice of the elementary formula does matter. For instance, in January 1999, the index formula used at the lower level of aggregation in the US consumer price index (CPI) calculations was changed to the ratio of geometric means of prices (Silver and Heravi 2007). The effect of this change was researched by the Bureau of Labor Statistics (2001) and it turned out that the change had caused a reduction of the annual rate of increase in the CPI of approximately 0.2 percentage points. Using the Boskin et al. (1996), estimates, according to which the effect of correcting a 1.1 percentage point overstatement in the CPI on federal debt was USD 1066.6 billion reduction by 2008 (see Boskin et al. (1996), we can conclude that the previously used elementary price index would generate a cumulative additional national debt from over-indexing the federal budget of approximately USD 200 billion (USD 1066.6 0.2/1.1 billion) over the twelve-year period up to the mid-1990s (see also Boskin et al. 1998).

In March 2013, the UK's Office for National Statistics (ONS) started to publish a new inflation index - RPIJ. This index is identical to the Retail Price Index (RPI), except it uses a geometric mean of price relatives (known as the Jevons index) rather than an

\footnotetext{
${ }^{1}$ Department of Statistical Methods, University of Lodz, ul. Uniwersytecka 3, 90-137, Lodz, Poland. Email: jacek.bialek@uni.lodz.pl.

Acknowledgments: The article is financed by the National Science Centre in Poland (grant no. 2017/25/B/HS4/00387). The article represents the author's personal opinion and does not necessarily reflect the view of Statistics Poland. I would like to thank the anonymous reviewers for their very helpful comments and inspiring remarks.
} 
arithmetic mean of price relatives (the Carli index). At that time, the UK Statistics Authority decided that, due to the potential upward bias in the Carli index, the old RPI would no longer be recognised as a national statistic (UK Statistics Authority 2013).

The above-mentioned decision was quite controversial. On the one hand, the new RPIJ gave a much lower rate of inflation than the RPI (the RPI gave an average inflation rate of $2.9 \%$ for the years 1998-2013, whereas the RPIJ gave 2.5\% (Levell 2015)). The use of the Jevons index in the CPI generated some mistrust in the official numbers in the United Kingdom. In other words, the CPI replaced the RPI for policy purposes, since the government replaced the RPI with the CPI for the indexation of state benefits, government pensions and tax thresholds (Levell 2015). On the other hand, due to the concern of the ONS about the Carli index's sensitivity to "price bouncing" and knowing that the Carli index failed some other important tests from the axiomatic price index theory (time reversal test, circularity - see Diewert (2012)), the ONS opted not to change the RPI, but rather to produce a new index. In January 2013, the ONS stated that the Carli index "did not meet international standards" (Office for National Statistics 2013). At present time, none of the 27 European Union countries makes use of the Carli index in their national price indices. Eurostat regulations do not allow the use of the Carli index in the construction of the Member States' Harmonised Index of Consumer Prices (HICP). There has been a general trend of replacing the Carli index with the Jevons or the Dutot formulas (Evans 2012). Some countries abandoned the Carli index formula in favour of other price indices over the last few decades, for example, Canada (in 1978), Luxemburg (in 1996), Australia (in 1998), Italy (in 1999), and Switzerland (in 2000). In 1996, in the United States, the Boskin Commission recommended that a Carli-like index that was used in the US CPI should be replaced by the Jevons index (Levell 2015).

There are many papers that compare the above-mentioned unweighted price index numbers. Early contributions of Eichhorn and Voeller (1976), Dalen (1992) and Diewert (1995) provide studies of properties of elementary indices from an axiomatic point of view. The differences between elementary indices, in terms of changes in the price variances, have been considered for sample indices by using Taylor approximations (see e.g. Dalen 1992; Diewert 1995; Balk 2005 for details). There are some papers that also compare the population elementary indices (Silver and Heravi 2007). The statistical approach, in which calculated elementary indices are treated as estimators of population indices, can be found in the following papers: Balk (2005), McClelland and Reinsdorf (1999), Dorfman et al. (1999) or Greenlees (2001). For instance, McClelland and Reinsdorf (1999) draw attention to the small sample bias in the case of the sample Jevons index as an estimator of its population counterpart. Finally, some authors, for comparisons, also use axiomatic and economic approaches (Diewert 1995; Levell 2015), or a sampling approach (Balk 2005), in which differences between elementary indices are explained with respect to the sampling design. The earlier literature, using the actual data underlying the consumer price index, has shown that the differences at the elementary aggregate level between the Dutot, Carli and Jevons indices can be quite substantial (see Carruthers, et al. 1980; Dalen 1994; Schultz 1995; Moulton and Smedley 1995).

In this article, we focus on only two elementary price indices, namely we consider the Jevons and Carli formulas. It has been shown (see Hardy et al. (1934) for details concerning inequalities for elementary mean values) that the difference between the Carli index and the 
Jevons index is bounded from below by the variance of the price relatives. In this article, we extend this result under the assumption that prices are described by a geometric Brownian motion (GBM). We confirm some commonly known facts (such as a negative bias of the Jevons sample price index) and we also obtain some new results, including approximations for variances and mean squared errors (MSEs) of the Jevons and Carli indices and their asymptotic behaviour. According to the author's knowledge, there is a lack of works in the literature that use stochastic models with continuous time to compare elementary indices. The advantages of this approach have been emphasised in the further part of the article (see Empirical Illustration: Case 2 and Conclusions) but please note that one of new possibilities is the potential comparison of the quality of sample price indices (as estimates) at any time points, not necessarily being directly observed. Moreover, we can predict expected values of the sample Jevons and Carli indices and their other statistical characteristics for future time moments under the assumption that the nature of price processes will not change. In particular, our approach provides the possibility of forecasting expected values or variances of these sample indices or determining the above-mentioned characteristics for compared moments in time for which we do not have direct data. To be more precise: we provide new approximations to the biases, variances and mean squared errors of these indices for the above-mentioned stochastic price process. It is shown that these approximations may strongly depend on the sample size and price volatilities, but this remark is not identical for both considered indices. For instance, it is shown that the sample Carli index is an unbiased estimator of the unweighted population parameter describing the price change and the sample Jevons index is an asymptotically unbiased estimator of the same parameter. There are some other practical conclusions that can be drawn from our research; for instance, that the expected value of the Jevons index is sensitive to price volatility only in the case of small sample sizes, while the expected value of the Carli index does not depend on price dispersion (see Tables 4 and 5).

The article is organised as follows: Section 2 presents unweighted Jevons and Carli indices. Section 3 starts from the introduction to the GBM price model and compares the above-mentioned elementary indices in this area. Section 4 presents two empirical illustrations of the previously discussed theoretical results: the first study concerns prices of tomatoes sold in some number of supermarkets, the second one considers prices of mountain bikes sold via the largest online e-commerce platform in Poland. Section 5 discusses the results from our simulation study and examines the influence of price volatility on differences between the discussed sample indices; Section 6 lists the main conclusions derived from both empirical and simulation studies.

\section{Unweighted Jevons and Carli Indices}

There are several elementary price indices in the literature (Von der Lippe 2007; Consumer Price Index Manual, CPI Manual 2004 chap. 20). In particular, we have the following formulas:

- the Carli price index (Carli 1804)

$$
P_{C}=\frac{1}{N} \sum_{i=1}^{N} \frac{p_{i}^{t}}{p_{i}^{0}},
$$


- and the Jevons price index (Jevons 1865)

$$
P_{J}=\prod_{i=1}^{N}\left(\frac{p_{i}^{t}}{p_{i}^{0}}\right) \frac{1}{N}=\frac{\prod_{i=1}^{N}\left(p_{i}^{t}\right)^{1 / N}}{\prod_{i=1}^{N}\left(p_{i}^{0}\right)^{1 / N}}=\frac{\exp \left[\frac{1}{N} \sum_{i=1}^{N} \ln \left(p_{i}^{t}\right)\right]}{\exp \left[\frac{1}{N} \sum_{i=1}^{N} \ln \left(p_{i}^{0}\right)\right]}
$$

where the time moment $\tau=0$ is considered as the basis, $N$ is the number of items observed at times 0 and $t, p_{i}^{\tau}$ denotes the price of the $i$-th item at time $\tau$. The Carli index is an arithmetic mean of price relatives (partial indexes), whereas the Jevons index is a geometric mean. As a consequence, these indices satisfy the classic inequality for arithmetic and geometric means

$$
P_{J} \leq P_{C}
$$

The difference between the Carli index and the Jevons index is bounded from below by the variance of the price relatives $D^{2}\left(p_{i}^{t} / p_{i}^{0}\right)$ (for details concerning inequalities for elementary mean values, see for example: Chapter II in Hardy et al. 1934):

$$
P_{C}-P_{J} \geq D^{2}\left(\frac{p_{i}^{t}}{p_{i}^{0}}\right),
$$

and thus the analogical inequality holds for their expected values. From the point of view of the axiomatic price index theory, the Jevons index seems to be better, that is, it satisfies the main tests (axioms), whereas the Carli index does not satisfy the time reversal test and circularity (Levell 2015). Price indices defined in Equations (1) and (2) can be treated as sample indices, since they are estimators of unknown real values of population indices. In particular, the sample Carli index (1), being an arithmetic mean of price relatives, is a consistent estimator of the population Carli index that can be expressed as follows:

$$
I_{C}=E\left[\frac{p^{t}}{p^{0}}\right]
$$

Similarly, the sample Jevons index (2), as a ratio of the exponents of two sample means of $\log$ prices (Silver and Heravi 2007), is a consistent estimator of the following population Jevons index, that is,

$$
I_{J}=\frac{\exp \left[E\left[\ln \left(p^{t}\right)\right]\right]}{\exp \left[E\left[\ln \left(p^{0}\right)\right]\right]}
$$

At this point, the reader needs a certain clarification. First of all, following the work of Silver and Heravi (2007), in the presented approach, population indices are unknown a priori values expressed by expected values of price relatives or log-prices. We consider here a homogeneous aggregate, and thus a random variable, that is, the price from the examined period or the base period, reflects the prices of items of the same product sold in a given period by different establishments. Assuming a specific probability distribution for the prices from the base and the analysed period, or, as in our article, adopting a specific stochastic process describing the price process in the whole examined period of time, we are able to determine population indices without referring to the size of the population 
(which in fact is difficult to determine in practice), and the determination of the parameters of this distribution or process is a technical issue (see Subsection 3.2). We want to estimate population indices using the sample indices described by Equations (1) and (2) determined on the basis of a drawn sample of $N$ matched items. In the literature, however, one can encounter a slightly different approach in which in the case of homogeneous aggregate, the target (or population) price index is the unit value index, and the population Jevons and Carli indices are defined analogously to Equations (1) and (2), where this time $N$ denotes the size of the population, and sample elementary indices are calculated on the basis of a random sample that constitutes a subset of the above-mentioned population (see e.g. Diewert 1995; Balk 2005). Secondly, in the quoted work of Balk (2005), the author considers two scenarios of obtaining the sample. The first scenario assumes the simple random sample drawn without replacement, which means that each element of the population has the same probability of being included in the sample. In the other scenario, the more "important" elements of the population have a larger probability of being included in the sample than the less important elements. As the author himself admits, both scenarios are "more or less representative of actual statistical practice". Nevertheless, this article implicitly assumes that the first scenario is implemented.

\section{Basic Statistics of the Carli and Jevons Sample Indices in the Stochastic Model}

\subsection{The GBM Price Model}

A geometric Brownian motion (GBM) (also known as exponential Brownian motion) is a continuous-time stochastic process in which the logarithm of the randomly varying quantity follows a Brownian motion (also called a Wiener process) with drift (see Oksendal 2003; Privault 2012). It is an important example of stochastic processes satisfying a stochastic differential Equation (SDE, see Subsection 3.2. for more details). In particular, the GBM model is used in mathematical finance to model stock prices in the Black-Scholes model (Privault 2012; Ross 2014) and, for instance, to model prices of derivatives (Hull 2018). In the literature, we can encounter many other applications of this model, for instance: modelling of unit prices of Open Pension Funds (Gajek and Kałuszka 2004; Białek 2013), modelling of oil prices (Meade 2010; Nwafor and Oyedele 2017), or modelling of electricity prices (Barlow 2002). The GBM price model can be also used for generalisations of the Divisia's approach in the price index theory (Białek 2015).

The main arguments for using the GBM price model are as follows:

(a) the expected returns (relative price changes) are independent of the value of the process (price), which is consistent with what we would expect in reality;

(b) the GBM process only assumes positive values, just like real commodity prices;

(c) the GBM process shows the same kind of 'roughness' in its paths as we see in real prices; and

(d) estimations of its parameters are relatively easy.

Nevertheless, the GBM model also has some drawbacks: (A) the price volatility is assumed constant in this model; (B) the GBM model does not take into account possible 
jumps of prices caused by unpredictable events or news (see the final remarks in Conclusions, Section 6) since the path in GBM model is continuous. However, due to the advantages of using the GBM price model presented above, we decided to apply it in our work. The application of more advanced models, being generalisations or extensions of the GBM model (see e.g. Kou 2002; Kühn and Neu 2008; You-Sheng and Cheng-Hsun 2011; Hong-Bae and Tae-Jun 2015), in the theory and practice of price indices is our future aim.

\subsection{Comparison of Carli and Jevons Indices Under the GBM Price Model}

In practice, unweighted indices (such as the Carli, Jevons or Dutot indices) are used at the lowest level of aggregation in the CPI measurement (Von der Lippe 2007). In general, the calculation of the Consumer Price Index (CPI) proceeds in two (or more) stages. In the first stage, elementary indices are estimated for the elementary expenditure aggregates of the CPI. In the second and subsequent stages of data aggregation, these elementary price indices are combined to obtain higher-level price indices using information on the expenditures on each of the elementary aggregates as weights (CPI Manual 2004 chap. 20). In other words, these higher-level price indices are aggregated further through expenditure-weighted averages into "sections" that, in turn, are aggregated into "groups" (Levell 2015).

Let us go back to the lowest level of aggregation. In the very first stage, in which the Office for National Statistics (ONS) does not have expenditure information, each matched item is observed in many monitoring points (sampled outlets) in the country. Thus, for a given homogeneous set of items, we have a corresponding set of monthly prices collected systematically for each month. As a consequence, most authors treat the above-mentioned set of prices from a given month as a realisation of one random variable and they assume a common price distribution for these prices (Silver and Heravi 2007; Levell 2015). In other words, elementary price indices concern rather a given kind of good (product) observed in many places (outlets), and thus described by a vector of different prices being realisations of the same random variable. According to the above-mentioned remark, let us assume one, common price distribution, that is, we assume that all price processes (representing the considered matched items) can be described by a geometric Brownian (Wiener) motion (GBM), also known as an exponential Brownian motion. To be more precise, we assume that the given $i-$ th price process satisfies the following stochastic differential equation

$$
d p_{i}^{t}=\alpha p_{i}^{t} d t+\beta p_{i}^{t} d W_{i}^{t},
$$

where the percentage drift $\alpha$ and the percentage volatility $\beta$ are constant, and $\left\{W_{i}^{t}: 0 \leq\right.$ $t<\infty, i=1,2, \ldots, N\}$ are independent Wiener processes. The solution for the stochastic differential Equation (7) is as follows (Oksendal 2003; Jakubowski et al. 2003):

$$
p_{i}^{t}=p_{i}^{0} \exp \left(\left(\alpha-\frac{\beta^{2}}{2}\right) t+\beta W_{i}^{t}\right),
$$

and we assume that all initial prices $p_{i}^{0}$ are deterministic. As a consequence, we obtain

$$
E\left(P_{i}^{t}\right)=\exp (\alpha t)
$$

and

$$
\operatorname{Var}\left(P_{i}^{t}\right)=\exp (2 \alpha t)\left[\exp \left(\beta^{2} t\right)-1\right]
$$


where $P_{i}^{t}$ is a $i$-th price relative and $\exp (\alpha t)$ is the (unknown) population price index that we want to estimate (Oksendal 2003; Jakubowski et al. 2003). Let us denote by $\mu_{t}=E\left(P_{i}^{t}\right)$ and by $\sigma_{t}^{2}=\operatorname{Var}\left(P_{i}^{t}\right)$ for any value of $i$. Let us note that the sample Jevons index can be expressed as follows:

$$
P_{J}=\prod_{i=1}^{N}\left(P_{i}^{t}\right)^{\frac{1}{N}}=\prod_{i=1}^{N} \exp \left(\frac{\alpha-\beta^{2} / 2}{N} t+\frac{\beta}{N} W_{i}^{t}\right)
$$

or equivalently

$$
P_{J}=\exp \left(\left(\sum_{i=1}^{N} \frac{\alpha}{N}-\frac{1}{2} \sum_{i=1}^{N}\left(\frac{\beta}{N}\right)^{2}\right) t+\sum_{i=1}^{N} \frac{\beta}{N} W_{i}^{t}\right) \exp \left(\frac{1}{2}\left(\sum_{i=1}^{N}\left(\frac{\beta}{N}\right)^{2}-\sum_{i=1}^{N} \frac{\beta^{2}}{N}\right) t\right)
$$

Let us denote by $\operatorname{vol}(t, \beta, N)$ a component connected with price volatilities, that is,

$$
\operatorname{vol}(t, \beta, N)=\exp \left(\frac{1}{2}\left(\sum_{i=1}^{N}\left(\frac{\beta}{N}\right)^{2}-\sum_{i=1}^{N} \frac{\beta^{2}}{N}\right) t\right)=\exp \left(\frac{1-N}{2 N^{2}} \beta^{2} t\right) .
$$

We have

$$
E\left(P_{J}\right)=\operatorname{vol}(t, \beta, N) \prod_{i=1}^{N} E\left[\exp \left(\left(\frac{\alpha}{N}-\frac{1}{2}\left(\frac{\beta}{N}\right)^{2}\right) t+\frac{\beta}{N} W_{i}^{t}\right)\right.
$$

From Equations (9) and (14), we obtain the following expected values of the sample Carli and Jevons indices:

$$
\begin{gathered}
E\left(P_{C}\right)=\frac{1}{N} \sum_{i=1}^{N} E\left(P_{i}^{t}\right)=\exp (\alpha t)=\mu_{t}, \\
E\left(P_{J}\right)=\operatorname{vol}(t, \beta, N) \exp \left(\frac{\alpha}{N} t\right)^{N}=\operatorname{vol}(t, \beta, N) \mu_{t} .
\end{gathered}
$$

The immediate conclusion from Equations (15) and (16) is a known fact that the sample Carli index is an unbiased estimator of the parameter $\mu_{t}$ and the sample Jevons index is a biased estimator of the same parameter, in which its bias is very small in practice (when the sample size $N$ is big) and it equals

$$
\operatorname{bias}\left(P_{J}\right)=E\left(P_{J}-\mu_{t}\right)=(\operatorname{vol}(t, \beta, N)-1) \mu_{t} .
$$

Moreover, from Equation (10) we obtain the variance of the sample Carli index, that is,

$$
\begin{aligned}
\operatorname{Var}\left(P_{C}\right) & =\frac{1}{N^{2}} \operatorname{Var}\left(\sum_{i=1}^{N} \frac{p_{i}^{t}}{p_{i}^{0}}\right)=\frac{1}{N^{2}} \sum_{i=1}^{N} \operatorname{Var}\left(P_{i}^{t}\right) \\
& =\frac{1}{N^{2}} \sum_{i=1}^{N} \exp (2 \alpha t)\left[\exp \left(\beta^{2} t\right)-1\right]= \\
& =\frac{1}{N} \mu_{t}^{2}\left[\exp \left(\beta^{2} t\right)-1\right]=\frac{\sigma_{t}^{2}}{N} .
\end{aligned}
$$


Let us note that from (11) it holds that

$$
\ln P_{J}=\left(\alpha-\frac{\beta^{2}}{2}\right) t+\frac{\beta}{N} \sum_{i=1}^{N} W_{i}^{t},
$$

and thus, since $\operatorname{Var}\left(W_{i}^{t}\right)=t$, we obtain

$$
\operatorname{Var}\left(\ln P_{J}\right)=\frac{\beta^{2}}{N^{2}} N t=\frac{\beta^{2} t}{N}
$$

From the Taylor's approximation rule, we know that $\ln (x) \approx x-1$ for $x \approx 1$, and thus we obtain that $\operatorname{Var}\left(\ln P_{J}\right) \approx \operatorname{Var}\left(P_{J}\right)$. Let us note that in practice the value of $\beta^{2} t$ will be close to zero, and thus using once again the Taylor's approximation rule (i.e., $\exp (x)-1 \approx x$ for small values of $x$ ) we obtain

$$
\operatorname{Var}\left(P_{i}^{t}\right)=\exp (2 \alpha t)\left[\exp \left(\beta^{2} t\right)-1\right] \approx N \mu_{t}^{2} \operatorname{Var}\left(P_{J}\right) .
$$

Thus, finally we obtain

$$
\operatorname{Var}\left(P_{J}\right) \approx \frac{\sigma_{t}^{2}}{\mu_{t}^{2} N} .
$$

The overall performance of an estimator can be summarised by its mean-squared error (MSE), which measures its expected squared deviation from the true population value of the parameter of interest. The MSE can be expressed as follows:

$$
\operatorname{MSE}(\hat{\theta})=E(\theta-\hat{\theta})^{2}=\operatorname{Var}(\hat{\theta})+\operatorname{bias}^{2}(\hat{\theta}),
$$

where $\hat{\theta}$ denotes a considered estimator of population parameter $\theta$. In our case, from Equations (17), (18) and (22), we obtain the following values of mean-squared errors of the sample Carli and Jevons indices:

$$
\begin{gathered}
\operatorname{MSE}\left(P_{C}\right)=\frac{\sigma_{t}^{2}}{N}, \\
\operatorname{MSE}\left(P_{J}\right) \approx \frac{\sigma_{t}^{2}}{\mu_{t}^{2} N}+(\operatorname{vol}(t, \beta, N)-1)^{2} \mu_{t}^{2} .
\end{gathered}
$$

Let us note that from Equation (13) we obtain for the fixed values of $t$ and $\beta$

$$
\lim _{N \rightarrow \infty} \operatorname{vol}(t, \beta, N)=\exp (0)=1 \text {. }
$$

In practice, the number $N$ is large, and thus we can use the following approximation:

$$
E\left(P_{J}\right)=\operatorname{vol}(t, \beta, N) \mu_{t} \approx \mu_{t} .
$$

From Equations (17) and (27), we obtain the following asymptotic property of the Jevons formula

$$
\lim _{N \rightarrow \infty} \operatorname{bias}\left(P_{J}\right)=\lim _{N \rightarrow \infty}(\operatorname{vol}(t, \beta, N)-1) \mu_{t}=0
$$

and, for big sample sizes, we obtain

$$
\operatorname{MSE}\left(P_{J}\right) \approx \frac{\sigma_{t}^{2}}{\mu_{t}^{2} N}+(\operatorname{vol}(t, \beta, N)-1)^{2} \mu_{t}^{2} \approx \frac{\sigma_{t}^{2}}{\mu_{t}^{2} N} .
$$


The immediate conclusion is that the bias of the sample Jevons index is negative (see also Greenlees 2001) and the estimator $P_{J}$ is asymptotically unbiased, see Equation (28). From Equations (24) and (29), we conclude that the MSEs of the sample Carli and Jevons indices can be reduced to zero by increasing the sample size. The MSEs of these sample indices are decreasing functions of the sample size and they tend to zero if $N \rightarrow \infty$. The following example shows that it is possible that $\operatorname{MSE}\left(P_{c}\right)-\operatorname{MSE}\left(P_{J}\right)$ is greater or smaller than zero, but in practice, this difference will be very small. Although our results, similarly to those obtained by Levell (2015), suggest that in general the ratio of the considered MSEs is greater or smaller than 1 depending on variances of prices or indices, from Equations (24) and (29), we obtain the following approximation for big sample sizes: $\operatorname{MSE}\left(P_{c}\right) / \operatorname{MSE}\left(P_{J}\right) \approx \mu_{t}^{2}$. Thus, in the case of increasing prices (i.e., when $\mu_{t}^{2}>1$ ), from the large data set, we can expect that $\operatorname{MSE}\left(P_{c}\right)>\operatorname{MSE}\left(P_{J}\right)$ - see also our results presented in Table 3.

\subsubsection{Example}

Let us consider a random sample connected with the given item observed in $N$ drawn monitoring points during the unit time interval. In other words, we observe a homogeneous group of identical items with different $N$ prices described in Equation (8). Let us denote the unknown population parameters at the end of the unit time interval as $\mu$ and $\sigma$. We take into consideration the following values of parameters of price processes: $\mu \in[0.8,1.2]$ and $\sigma \in[0,0.2]$. Let us denote by $\Delta=\operatorname{MSE}\left(P_{c}\right)-\operatorname{MSE}\left(P_{J}\right)$, where MSEs of indices are defined in (24) and (25). Differences $\Delta$ (as functions of parameters $\mu$ and $\sigma$ ) calculated for $N \in\{5,10,50,500\}$ are presented in Figure 1 .

a) $N=5$
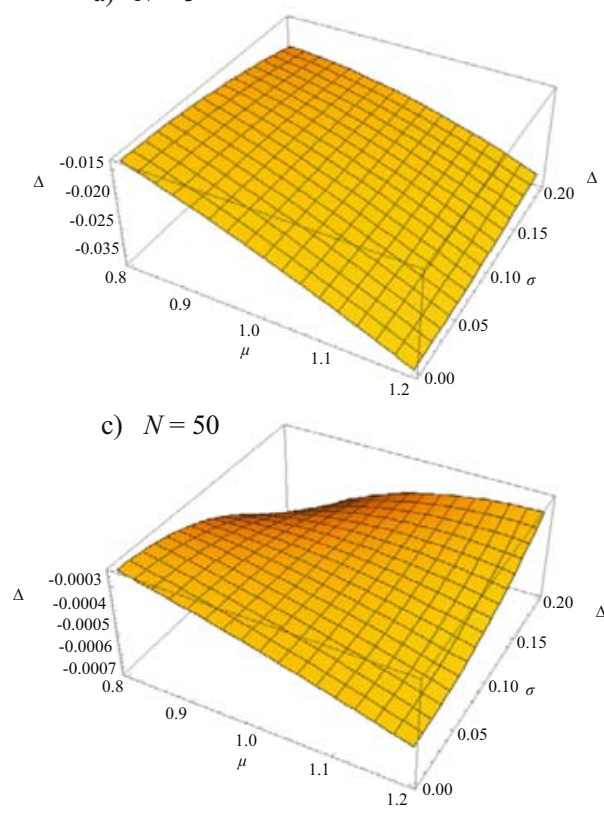

a) $N=10$

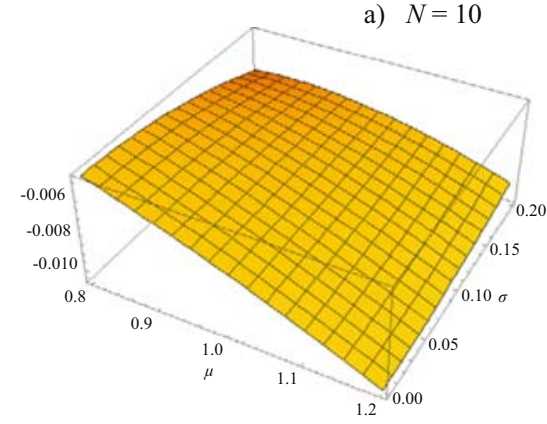

d) $N=500$

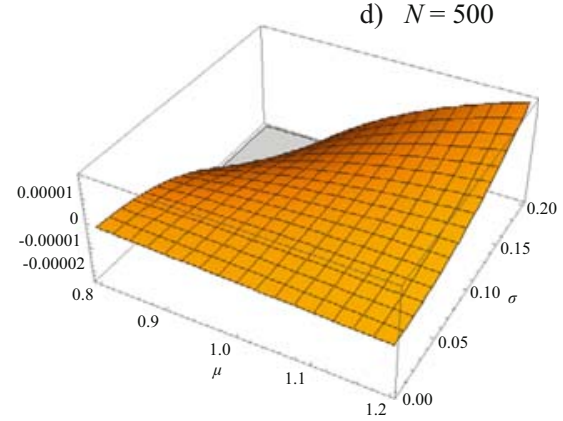

Fig. 1. Differences between MSEs of indices ( $\Delta)$ depending on $\mu, \sigma$ and $N$. 
Based on Figure 1, we can conclude that the difference $\operatorname{MSE}\left(P_{c}\right)-\operatorname{MSE}\left(P_{J}\right)$, as a function of parameters of price processes, is negative as a rule. Nevertheless, when the sample size is big and approximations Equations (27) and (29) start to work, the MSE of the sample Carli index is minimally bigger (smaller) than the MSE of the Jevons index if $\mu>1$ (respectively $\mu<1$ ).

\section{Empirical Illustration}

\subsection{Case 1}

As it was mentioned above, in practice, at the lowest level of aggregation we collect prices of the considered group of items, that is, each item is observed in many monitoring (sales) points in the country. Let us suppose we observe an item number $i_{0}$ during the time interval $[0, t]$ and we have $N$ sample prices of this item at time $0\left(p_{i_{0} k}^{0}: k=1,2, \ldots, N\right)$ and $N$ sample prices of this item at time $t\left(p_{i_{0} k}^{t}: k=1,2, \ldots, N\right)$. We obtain $N$ sample price relatives $\left(P_{i_{0} k}^{t}=p_{i_{0} k}^{t} / p_{i_{0} k}^{0}: k=1,2, \ldots, N\right)$ and, although we assume that prices are described by a geometric Brownian motion, we do not know the real values of the drift $\alpha$ and the volatility $\beta$ in the price population. In this article, we propose using very simple estimators of $\alpha$ and $\beta$ that are an immediate consequence of the fact that the mean of observed price relatives should be approximated by $\exp (\alpha t)$ and the standard deviation of observed price relatives should be approximated by $\exp (\alpha t) \sqrt{\exp \left(\beta^{2} t\right)-1}$ (under assumptions of the GBM model). These estimators are as follows:

$$
\begin{gathered}
\hat{\alpha}=\frac{\ln \bar{P}_{i_{0}}^{t}}{t} . \\
\hat{\beta}=\sqrt{\frac{\ln \left\{\exp \left[2\left(\ln S_{i_{0}}^{t}-\hat{\alpha} t\right)\right]+1\right\}}{t} .} .
\end{gathered}
$$

where $\bar{P}_{i_{0}}^{t}$ and $S_{i_{0}}^{t}$ denote the arithmetic mean and the standard deviation of price relatives $\left(P_{i_{0} k}^{t}: k=1,2, \ldots, N\right)$ at time $t$. Estimators (30) and (31) are quite effective for the number of monitoring points over 100. Having estimated parameters $\alpha$ and $\beta$, we can estimate $\operatorname{vol}(t, \beta)$ function, and thus we can approximate $\operatorname{bias}\left(P_{J}\right)$ or $\operatorname{MSE}\left(P_{J}\right)$ (see Equations (28) and (29)). Treating values of $\bar{P}_{i_{0}}^{t}$ and $S_{i_{0}}^{t}$ as good enough estimates of $\mu_{t}$ and $\sigma_{t}$ we can approximate $\operatorname{Var}\left(P_{C}\right), \operatorname{Var}\left(P_{J}\right)$ and $M S E\left(P_{C}\right)$ (see Equations (18), (22) and (24)). We apply this observation to the real data set connected with prices of $1 \mathrm{~kg}$ of tomatoes in Poland. We collect data about these prices from $N=115$ Polish supermarkets observed during the interval: 01.12.2017 - 19.01.2018 (for convenience, we normalise the interval to $[0,1]$ ).

In this experiment, we are going to estimate parameters of the GBM model using only the first and the last time moments of observations, that is, we compare only prices from 01.12.2017 and from 19.01.2018, since the interval is very short, and we use Equations (30) and (31) for estimation of these parameters. In a more practical case (see, for instance, Subsection 4.2), we take into account all time moments from the considered interval for the estimation purpose. Nevertheless, this time we want to verify whether we can still obtain good approximations of considered price indices while limiting our analysis to only two compared time moments (the base one and the current one). Our hypothesis states that 
Equations (15) and (16) may approximate the real value of price indices effectively even if estimates of parameters $\alpha$ and $\beta$ are based on only the first and the last moment of a short time interval (the only condition is a lognormal price distribution). After calculations, we obtain $\hat{\alpha}=0.18912$ and $\hat{\beta}=0.22629$. The real mean price process and sample realisation of the corresponding geometric Brownian motion are presented in Figure 2. We are aware of the fact that while estimating the above-mentioned parameters, none of internal time moments were used, and thus the theoretical price movements (see Figure $2 \mathrm{~b}$ ) may not be fitted to the empirical ones perfectly (Figure 2a). Nevertheless, we evaluate the fit of the GBM model to the data by determining at least simple statistics such as the Root Mean Squared Error (RMSE) or the Mean Absolute Percentage Error (MAPE). Our results $(R M S E=0.0228, M A P E=5.1938 \%)$ are acceptable, which may be a consequence of a lognormal distribution of price relatives. In fact, if the GBM model is a good proxy for price dynamics, then equivalently the price relatives, calculated for any fixed current time moment $t$, are well-described by a lognormal distribution (in our case, the KolmogorovSmirnov test of log-normality of price relatives $p_{i k}^{1} / p_{i k}^{0}$ returns $p$-value equals 0.112$)$. The empirical distribution of observed price relatives $\left(P_{i k}^{1}=p_{i k}^{1} / p_{i k}^{0}: k=1,2, \ldots, 115\right)$ is presented in Figure 3. Estimates for the Carli and Jevons indices (the day of 01.12.2017 is fixed as a base time period), their sample variances and MSEs are presented in Table 1.

Our hypothesis is confirmed, that is, in the case of lognormal price relatives, Equations (15) and (16) may approximate the real value of price indices effectively, even if estimates of model parameters $\alpha$ and $\beta$ are based on only the first and the last moment of a short time interval. Moreover, it can be observed that the difference between the observed Jevons and Carli indices is quite big due to the fact that the price volatility is large. Obviously, the sample Carli index is unbiased, while the bias of the sample Jevons index is negative and equals -0.0355 . As once could expect, the bias of the Jevons index is crucial since the analysed price processes have strong fluctuations (see Equations (13) and (17)). In the analysed case, the variance and the coefficient of variation of the sample Carli price index are bigger than those calculated for the Jevons index. Please note that the precision of estimation of the expected value could be better in the case of the Jevons formula (the estimation is perfect for the Carli price index), that is, we have a theoretical value 1.2079 versus the empirical one: 1.1724 . One of the reasons for this is the high price volatility, as the $\beta$ parameter is above 0.2 . The second reason, mentioned earlier, is that the estimation

a) The real mean prices of tomatoes

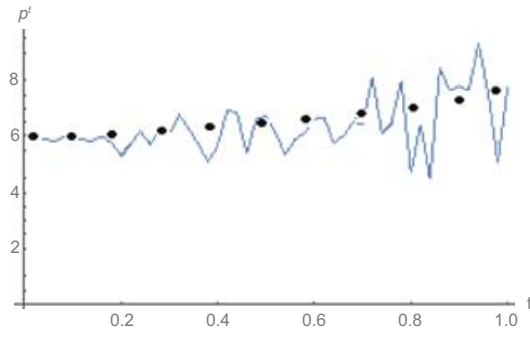

b) Sample realisation of the corresponding (continuous line) and some of fitted values - GBM price model

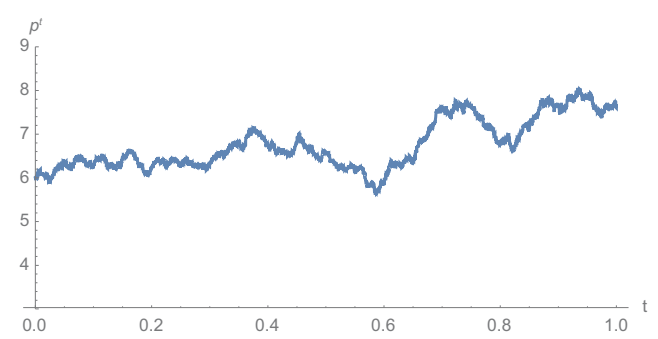

Fig. 2. Empirical and theoretical tomato price processes (Poland, 01.12.2017 - 19.01.2018). 


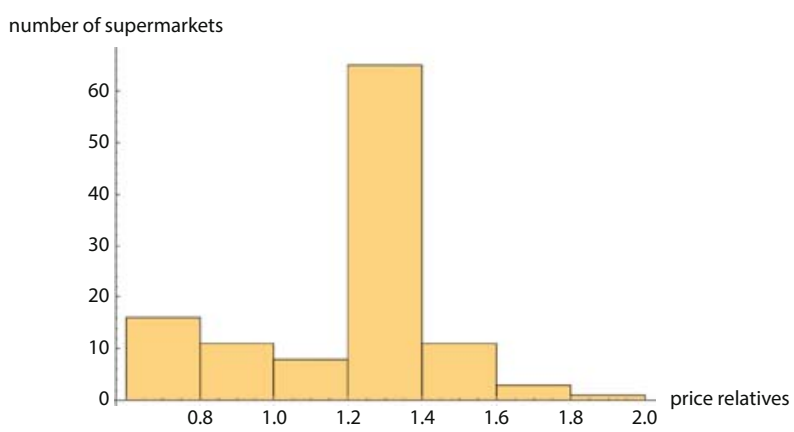

Fig. 3. The empirical distribution of observed tomato price relatives.

Table 1. Basic characteristics of the sample Jevons and Carli indices.

\begin{tabular}{lcc}
\hline Characteristics & Jevons index & Carli index \\
\hline Observed index value & 1.1724 & 1.2082 \\
Expected value $(*)$ & 1.2079 & 1.2082 \\
Standard deviation $(*)$ & 0.0211 & 0.0258 \\
Coefficient of variation $(*)$ & 0.0174 & 0.0213 \\
\hline
\end{tabular}

(*) Values obtained under the GBM price model for $\hat{\alpha}=0.18912$ and $\hat{\beta}=0.22629$.

of the GBM model parameters was made only for two time moments. When we have long time series and the model fit to the data is better, then we can also expect better approximations of the characteristics of the considered indices (see Case 2). It seems, therefore, that the discussed approach and method of estimation can be used only for very short time intervals and with the log-normality of price distribution.

\subsection{Case 2}

Case 1 concerns a situation in which we only have price observations of an item for two compared periods. In Case 1, we assumed that the process that allowed prices to go from the known state at the moment 0 to the known (observed) state at the moment $t$ was a geometric Brownian motion. Let us add that we made this assumption without any special possibility of its verification, or - due to only two moments of observation - without the possibility of assessing the fit of the GBM model to reality, and the estimators of the model parameters (30) and (31) were determined in a heuristic manner. Much more practical is a situation in which we have a long time series describing prices, and then we estimate the parameters $\alpha$ and $\beta$ using the GBM model dedicated estimators (usually Maximum Likelihood Estimators), and finally, we evaluate the fit of the GBM model to the data by determining at least simple statistics such as MAE, MAPE, RMSE, and so on. Please note that every time we calculate the level of the fit of GBM models or sample indices (based on the GBM model) we compare expected values of these stochastic processes to empirical observations. If the researchers become convinced that the GBM model describes the behaviour of prices well, then they have a basis for using the theoretical results obtained in Section 3. We will demonstrate this behaviour on the example of real data regarding the 
prices of mountain bikes sold in 2018 via allegro.pl, which is one of the largest online ecommerce platforms in Poland. To be more precise: we used weekly data on mountain bikes sold from 01.01. 2018 to 31.12.2018 (48 observations), and for demonstration purposes we decided to take into consideration $N=6$, the most popular in the considered time interval, mountain bike models with a tyre diameter of 28 inches (Denver, Davos, Indiana, Shimano, Romet, and Kross). Data were filtered and aggregated (we used unit values aggregated to one week as prices) by using a special tool provided by allegro.pl, that is, the TradeWatch (https://www.tradewatch.pl/index.jsf). After aggregating, we normalised each time series by dividing prices by the first observed price and we normalised the time interval of observations to $[0,1]$, that is, we set partial indices $\tilde{p}_{i}^{t}=p_{i}^{t} / p_{i}^{0}$ and time moments $t_{k}=\frac{k-1}{47}$ for $k=1,2, \ldots, 48$, and thus we obtained $\tilde{p}_{i}^{0}=1$, $t_{1}=0$ and $t_{48}=1$. For each normalised price process, the parameters $\alpha$ and $\beta$ of the GBM model were estimated by using the following unbiased estimators (Privault 2012):

$$
\begin{gathered}
\hat{\alpha}_{i}=\frac{1}{T-1} \sum_{k=1}^{T-1} \frac{1}{t_{k+1}-t_{k}}\left(\frac{p_{i}^{t_{k+1}}-p_{i}^{t_{k}}}{p_{i}^{t_{k}}}\right), \\
\hat{\beta}_{i}=\sqrt{\frac{1}{T-2} \sum_{k=1}^{T-1}\left(\frac{p_{i}^{t_{k+1}}-p_{i}^{t_{k}}}{p_{i}^{t_{k}}}-\hat{\alpha}_{i}\left(t_{k+1}-t_{k}\right)\right)^{2}},
\end{gathered}
$$

where $i=1,2, \ldots, 6$ and $T=48$. We also matched the GBM model for the time series of average bike prices, for the time series $\left\{\bar{p}_{t_{1}}, \bar{p}_{t_{2}}, \ldots, \bar{p}_{t_{48}}\right\}$ where $\bar{p}_{t_{k}}$ denotes the arithmetic mean of normalised prices of considered mountain bikes sold during the $k$ th week. For the above-mentioned purpose, we used estimators with an analogical form to those defined in Equations (32) and (33), namely

$$
\begin{gathered}
\hat{\alpha}_{T}=\frac{1}{T-1} \sum_{k=1}^{T-1} \frac{1}{t_{k+1}-t_{k}}\left(\frac{\bar{p}_{t_{k+1}}-\bar{p}_{t_{k}}}{\bar{p}_{t_{k}}}\right), \\
\hat{\beta}_{T}=\sqrt{\frac{1}{T-2} \sum_{k=1}^{T-1}\left(\frac{\bar{p}_{t_{k+1}}-\bar{p}_{t_{k}}}{\bar{p}_{t_{k}}}-\hat{\alpha}_{T}\left(t_{k+1}-t_{k}\right)\right)^{2},}
\end{gathered}
$$

where $k=1,2, \ldots, 48$ and $T=48$. Justification for matching the GBM model to the mean price process can be as follows:

(1) the fixed time moment prices described by the GBM model are log-normally distributed,

(2) the sum of log-normally distributed variables can be approximated by a lognormal distribution quite effectively (see Fenton-Wilkinson (FW) approximation: Fenton 1960; Cobb et al. 2012),

(3) it is easy to prove that if $X$ is $\log$-normally distributed, then $c X$ is also $\log$ normally distributed for any positive $c$, in particular for $c=1 / N$. 
Figure 4 presents observed (normalised) price processes and corresponding estimated price processes obtained from the matched GBM models (for each mountain bike case, information about estimated parameters $\alpha$ and $\beta$, as well as the level of RMSE is added below the right graph). Figure 5 presents the observed (normalised) average price process and the corresponding matched GBM model. The results obtained $\left(\hat{\alpha}_{T}=0.1737\right.$ and $\hat{\beta}_{T}=0.006636$ with a low level of $R M S E=0.0053$ ) allowed us to determine the expected values, standard deviations and coefficient of variations of the sample Carli and Jevons indices under the GBM price model (theoretical results are presented in Section 3). The

bike 1

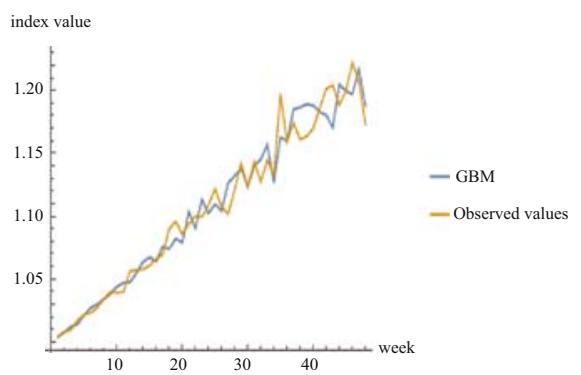

$\hat{\alpha}_{1}=0.19784 ; \hat{\beta}_{1}=0.01413 ; R M S E=0.01607$

bike 3

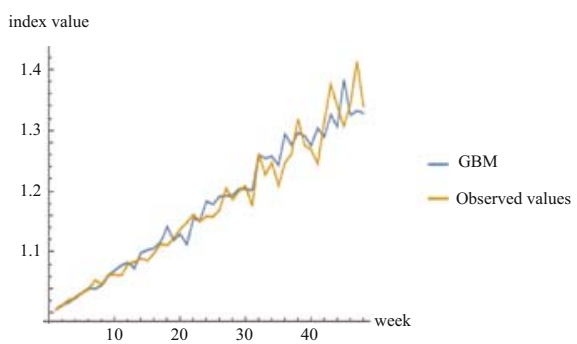

$\hat{\alpha}_{3}=0.30257 ; \hat{\beta}_{3}=0.0242979 ; R M S E=0.02457$

bike 5

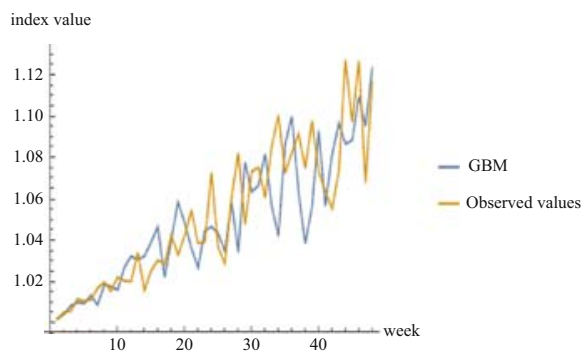

$\hat{\alpha}_{5}=0.09760 ; \hat{\beta}_{5}=0.02015 ; R M S E=0.02853$ bike 2

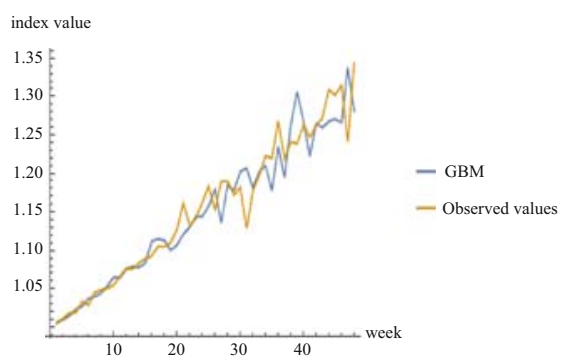

$\hat{\alpha}_{2}=0.27233 ; \hat{\beta}_{2}=0.02251 ; R M S E=0.02155$

bike 4

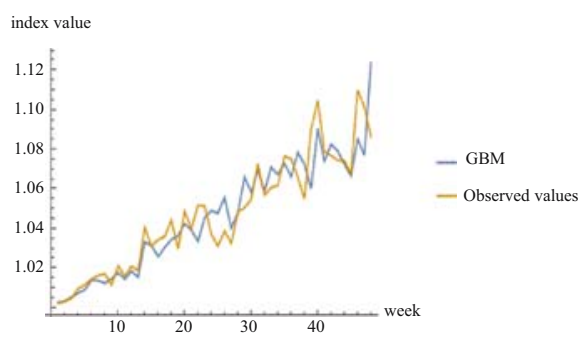

$\hat{\alpha}_{4}=0.09075 ; \hat{\beta}_{4}=0.01192 ; R M S E=0.01178$

bike 6

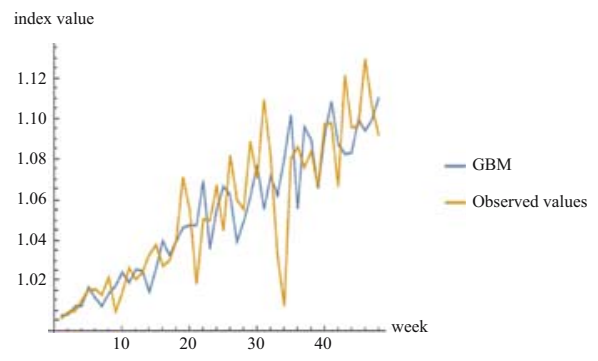

$\hat{\alpha}_{6}=0.10075 ; \hat{\beta}_{6}=0.2306 ; R M S E=0.02146$

Fig. 4. Observed normalised bike prices (Observed values) and sample realisations of matched GBM price models (GBM). 


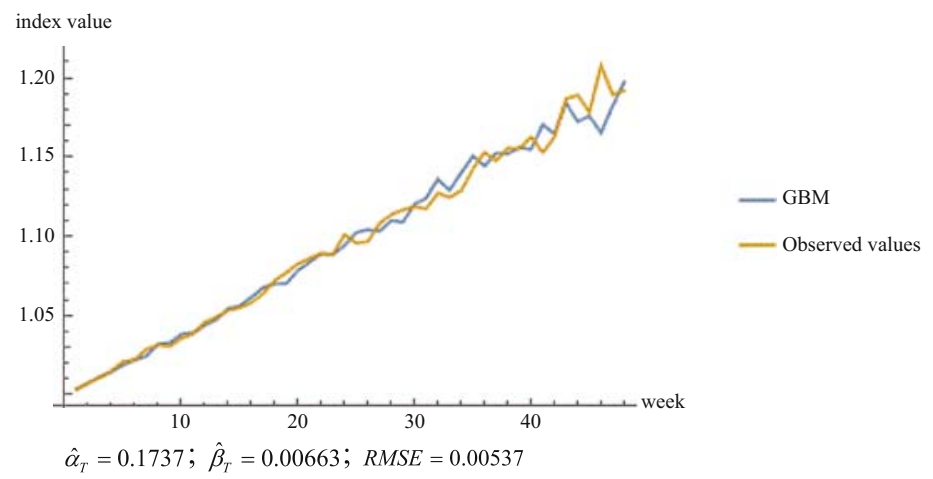

Fig. 5. Observed average normalised price process (Observed values) and sample realisation of the matched GBM price model (GBM).

results for the expected values of the sample indices obtained under the GBM model were then compared to the sample index values determined with Equations (1) and (2) based on a sample of all prices. All the results are presented in Figure 6 and Table 2. The small differences that we can observe in this comparison suggest not only that the adopted price

\section{Jevons index}

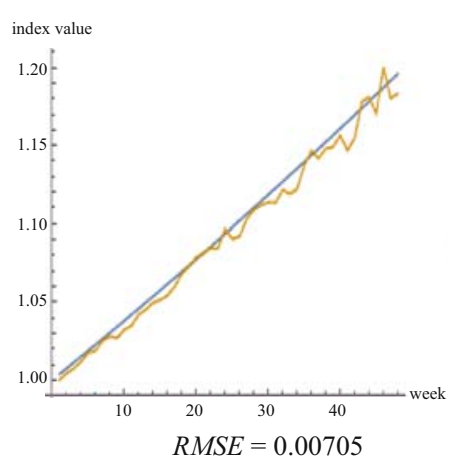

\section{Carli index}

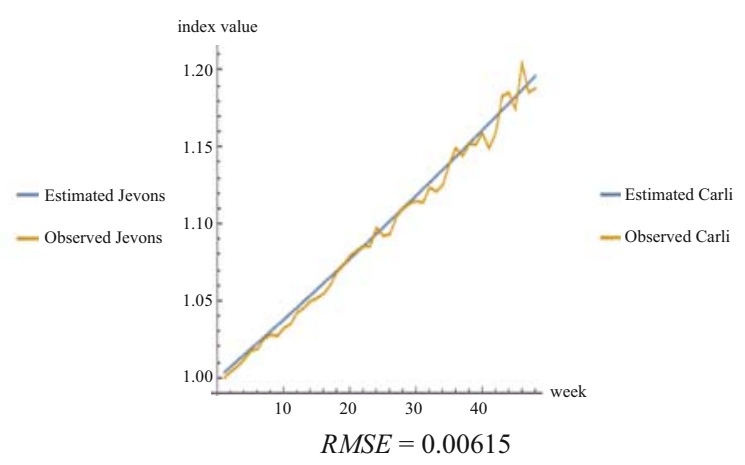

Fig. 6. Comparison of expected values of the Jevons and Carli indices with the empirical ones (the first week is the fixed base time period).

Table 2. Basic characteristics of the sample Jevons and Carli indices.

\begin{tabular}{lcc}
\hline Characteristics & Jevons index & Carli index \\
\hline Observed index value & 1.18319 & 1.18791 \\
Expected value $(*)$ & 1.18969 & 1.18970 \\
Standard deviation $(*)$ & 0.00271 & 0.00322 \\
Mean squared error $(\mathrm{MSE})(*)$ & $7.32636 \cdot 10^{-6}$ & $1.03883 \cdot 10^{-5}$ \\
\hline
\end{tabular}

(Indices calculated for the whole time interval, that is, the last week is compared to the first week).

(*) Values obtained under the GBM price model for $\hat{\alpha}_{T}=0.1737 ; \hat{\beta}_{T}=0.00663$. 
model is correct and its parameters have been properly determined, but also indicate the practical value of the theoretical results obtained. In practice, the estimation of parameters $\alpha$ and $\beta$ for a sufficiently long time series of prices (for which most probably $\hat{\alpha}_{T} \approx \hat{\alpha}_{T+1}$ and $\hat{\beta}_{T} \approx \hat{\beta}_{T+1}$ ) would allow for making short-term accurate predictions for the values of the elementary indices discussed (e.g. for one period ahead, i.e., for $t=T+1$ ). Similarly, retrospectively, it would be possible to make an accurate forecast of price changes (more precisely: to determine the expected sample indices in accordance with Equations (15) and (16)) between the time periods $m_{1}, m_{2} \in\left(t_{1}, t_{T}\right)$ which are of interest to the researcher, but for which direct price observations have not been made. For example, in the case of the analysed homogeneous group of mountain bikes, the forecasted (expected) price change after 197 days since its distribution (i.e., for $t=197 / 365=0.5397)$ is $E\left(P_{C}\right)=1.09829$, $E\left(P_{J}\right)=1.09828$.

\section{Simulation Study}

According to considerations from Section 4, we consider here a single item with prices described by a geometric Brownian motion (8) sold in $N_{m}$ places (outlets) and observed in $N$ sample monitoring points. We intend to compare expected values, biases and MSEs of the sample Jevons and Carli indices for different sample sizes. The population of $N_{m}$ prices is generated in four cases: Case 5.1 with the drift $\alpha=0.1$ and the volatility $\beta=0.03$ (increasing prices, low volatility), Case 5.2 with $\alpha=0.1$ and $\beta=0.3$ (increasing prices, high price volatility), Case 5.3 with $\alpha=-0.1$ and $\beta=0.03$ (decreasing prices, low price volatility) and Case 5.4 with $\alpha=-0.1$ and $\beta=0.3$ (decreasing prices, high price volatility). Our simulation procedure is as follows:

Step 1) we generate a population of $N_{m}=10,000$ prices described in Equation (8) for $t=1$ (without loss of generality, we assume that $p_{i}^{0}=1$ and thus the generated price $p_{i}^{1}$ determines the price relative $p_{i}^{1} / p_{i}^{0}$ );

Step 2) we draw a sample of $N$ prices $r=1,000$ times, whereas we consider $N \in\{10,100,1,000\}$;

Step 3) we calculate the sample Jevons and Carli indices for each $r$ th repetition and their distances to the theoretical expected price change $\mu=\exp (\alpha)$;

Step 4) we calculate expected values, standard deviations and coefficients of variation, as well as "empirical" biases and MSEs of the generated sample Jevons and Carli indices and we compare these results to theoretical ones obtained from the formulas presented in Subsection 3.2.

The populations of price relatives for Cases 5.1-5.4 are presented graphically in Figure 7. The sample realisations of price processes described by Cases 5.1-5.4 are presented in Figure 8. Empirical biases and MSEs of the sample Jevons and Carli indices and the analogous theoretical values are presented in Table 3. 
Case $5.1(\alpha=0.1$ and $\beta=0.03)$

number of obs.

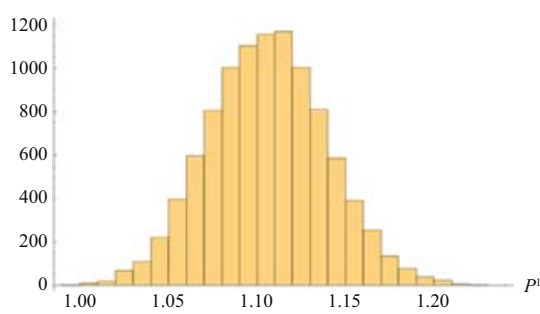

mean value: 1.10518 , standard deviation: 0.03340 , coefficient of variation: 0.03022 ;

Case $5.3(\alpha=-0.1$ and $\beta=0.03)$

number of obs.

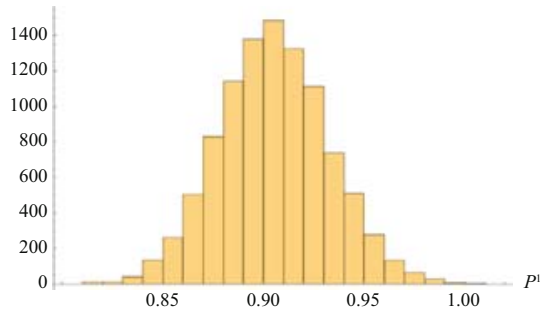

mean value: 0.904967 , standard deviation: 0.02725 , coefficient of variation: 0.03011 ;
Case $5.2(\alpha=0.1$ and $\beta=0.3)$

number of obs.

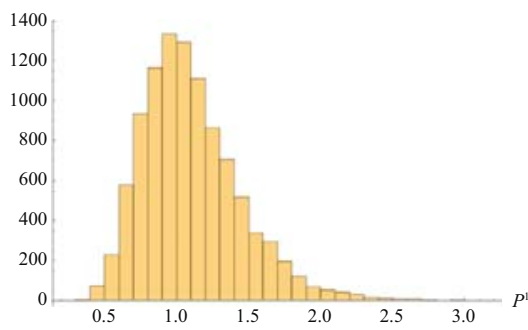

mean value: 1.10824 , standard deviation: 0.34359 , coefficient of variation: 0.31003

Case $5.4(\alpha=-0.1$ and $\beta=0.3)$

number of obs.

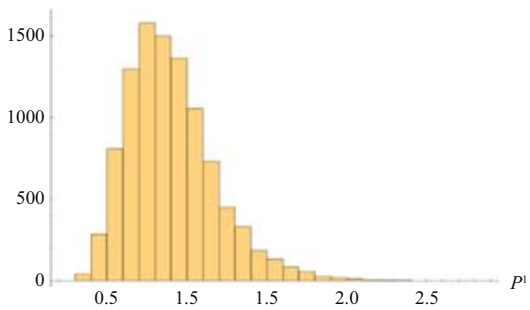

mean value: 0.904837 , standard deviation: 0.27767 , coefficient of variation: 0.30687

Fig. 7. Histogram and XY-plot for $N_{m}=10,000$ generated price relatives (population).

Case 5.1. $(\alpha=0.1$ and $\beta=0.03)$

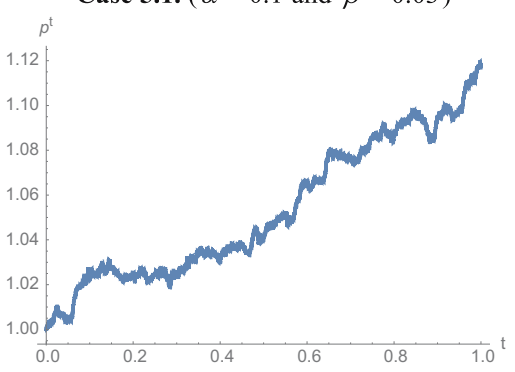

Case 5.3. $(\alpha=-0.1$ and $\beta=0.03)$

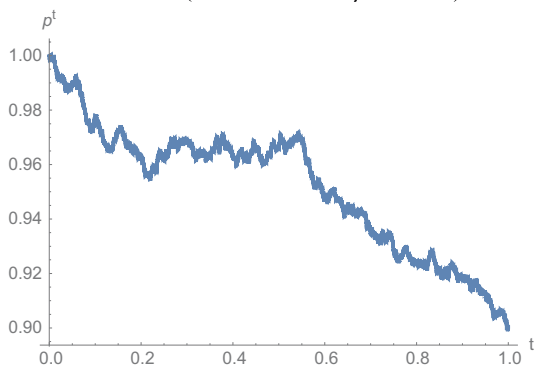

Case 5.2. $(\alpha=0.1$ and $\beta=0.3)$

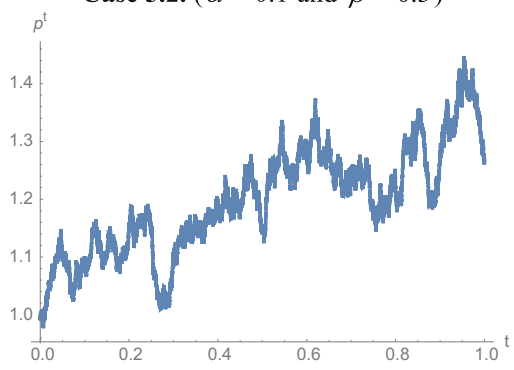

Case 5.4. $(\alpha=-0.1$ and $\beta=0.3)$

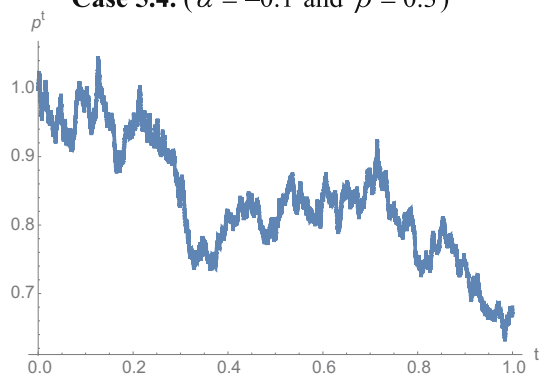

Fig. 8. Sample realisation of price processes from Cases 5.1-5.4. 


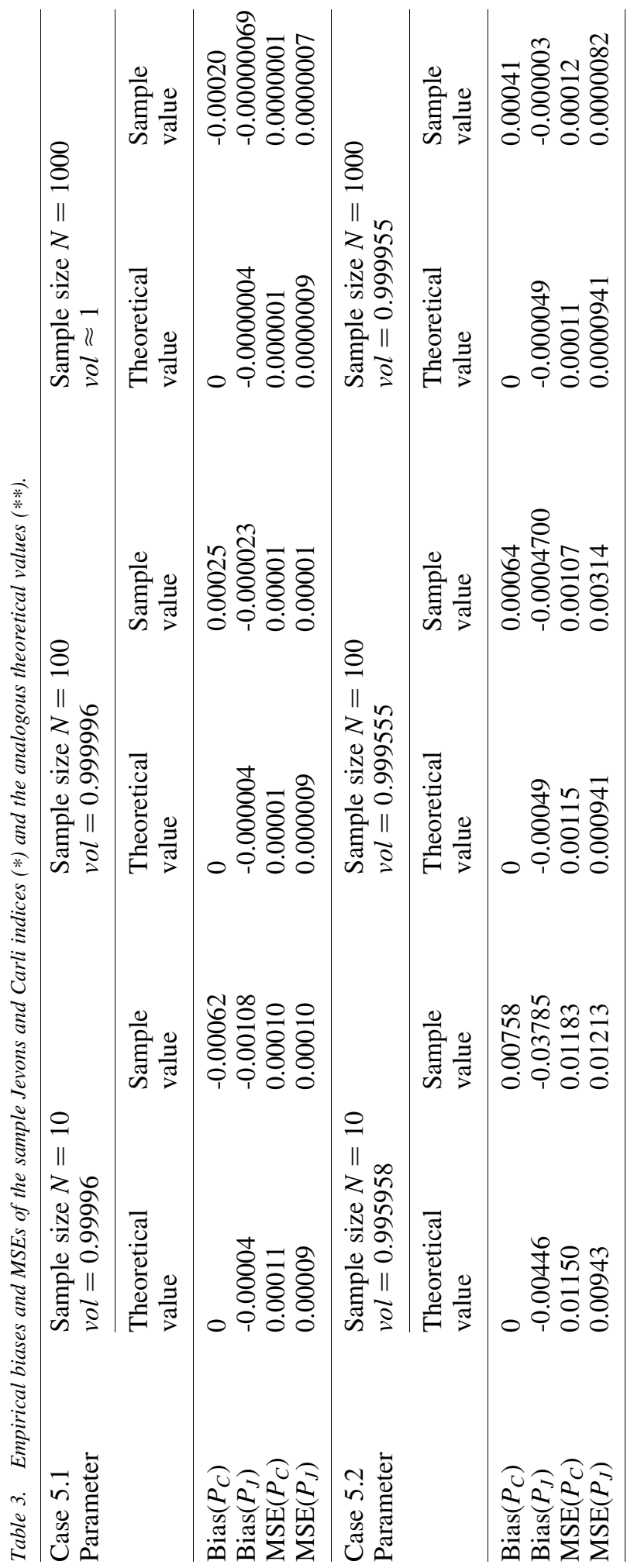




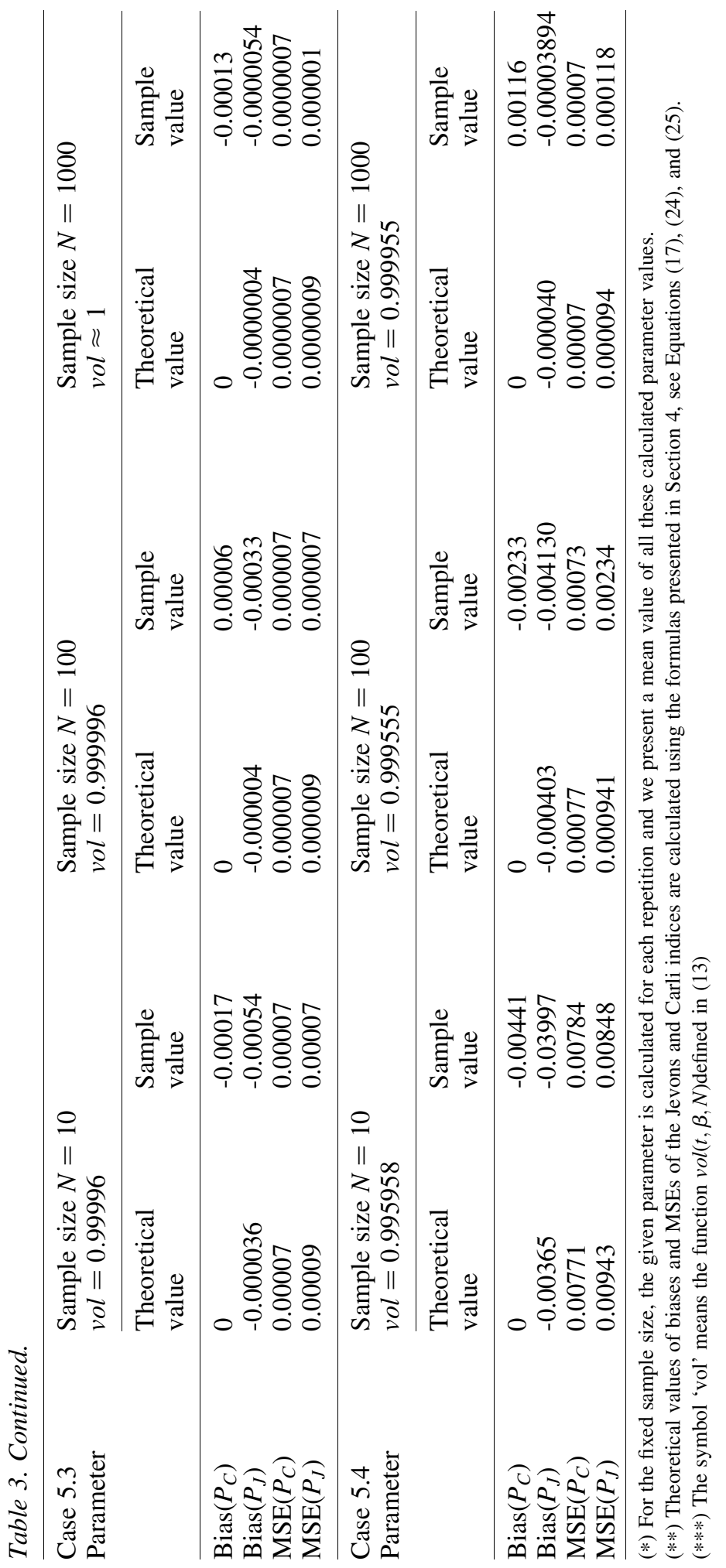




\section{Conclusions}

There has been a general trend of replacing the Carli index with the Jevons or the Dutot formulas, and most papers recommend the Jevons index rather than the Carli index. In this article, we show some similarities and differences in the practical use of these indices.

In the Simulation, we generate a price population and we compare biases and mean standard errors of the sample Jevons and Carli indices under the assumption that price processes have the same drifts and volatilities. The aim of the simulation is to verify how effective approximations (24) and (29) are. In Cases 5.1 and 5.3 with low price volatility, theoretical values of biases and MSEs of indices seem to be comparable with those obtained from samples (see Table 3). In Cases 5.2 and 5.4. with high price volatility, we also obtain a satisfying estimation of biases and mean standard errors of the considered sample indices in comparison with their theoretical values (see Table 3). Approximations (22) and (29) seem to work well and we can draw some general, partly already known, conclusions. Firstly, the sample Jevons index has a negative (and as a rule small) bias. It is proven, however, that the sample Jevons index is asymptotically unbiased under the GBM price model. Secondly, the difference between MSEs of the Jevons and Carli estimates depends not only on price volatilities but also, for the fixed level of price volatilities, it may depend on the sample size (see Table 3). Our simulation confirms theoretical results that the MSE of the sample Carli index is greater than the MSE of the Jevons index in the case of a big sample size and increasing prices. This relation will change its direction if prices start to decrease. We should be aware of one more thing - although differences between expected values, variances and MSEs of the sample Jevons and Carli indices seem to be negligible, that observation concerns only one considered item. In practice, the CPI basket consists of hundreds or thousands of items (depending on the country) and the choice of the elementary formula may have serious consequences for the final CPI calculations. In the literature, the above-mentioned source of the CPI bias is called the elementary formula bias (White 1999). Our other simulations (which are not presented here) also confirm results obtained in Equations (15), (16), (18), and (22). Theoretical values of the expected value, as well as the standard deviation and coefficient of variation of the considered sample elementary indices, are almost identical with those obtained via the simulation study for each sample size. And finally, in the case of both indices and all the considered cases, we can observe that by increasing the size of the sample ten times, we reduce their standard deviation (and coefficient of variation) by approximately three times.

The empirical study (see Section 4) confirms the usefulness of the theoretical results obtained. The study from Case 1 took into account the price of tomatoes in Poland, which is characterised not only by a high fluctuation of value in our climatic conditions, but also by seasonality. Due to the high volatility of prices, the obtained differences between the sample Jevons and Carli indices are noticeable, which once again confirms that the choice of the elementary formula of the index is extremely important in the CPI measurement. In our empirical study, the sample Carli price index has a larger variance but a smaller MSE than the sample Jevons index. As shown by our theoretical considerations and Example 1 (see Figure 1), this is not a general regularity. Interestingly, while using the Dalen's approximation of the Jevons index variance (Dalen 1999), it can be concluded that the 
approximate variance of the Jevons index will always be smaller than the variance of the Carli index. However, if we consider the formulas for the variance obtained in the article, see Equations (18) and (22), this relationship seems to be true, but only in the case of rising prices (i.e., when $\alpha>0$ then $\mu_{t}>1$ ). The empirical study presented in Case 2 concerns prices of six models of mountain bikes that were sold in 2018 (data on transactions were downloaded from allegro.pl). We match the GBM price model to this data set successfully (see Figures 4-6) and, after estimating the parameters of the model for average prices, we are able to: (a) calculate expected values, standard deviations and coefficients of variation of the sample Carli and Jevons indices (see Table 2); (b) forecast future values of these indices under the assumption that the model's parameters do not change rapidly over time; (c) calculate the historical values of these indices even for time moments from which there was no information about prices. Possibilities (b) and (c) are the main advantages of using continuous time price models such as the discussed GBM model.

To sum up, although the test and economic approaches both seem to favour the Jevons index over the Carli index (the Carli index fails time reversal test and circularity), the statistical approach does not provide clear, general guidance. The author's opinion in this respect coincides with the results obtained by Levell (2015). The problem of choosing between these indices is still open - and it is troubling that, for example, under our model assumptions, the expected value of the Jevons index is very strongly dependent on the level of price volatility.

Now some final remarks: the GBM process has a number of desirable properties when it comes to modelling prices (that are currently described in Subsection 3.1). It is shown in the article, by using an empirical example, that price movements are well-described by this process and, by using simulation exercises, that the approximations of the variance, bias, and MSE of these indices are accurate. The article also shows that given this stochastic process, the difference between the Jevons and Carli indices depends specifically on the volatility of prices. Without knowledge of the exact process followed by prices, we can otherwise only say that the difference is bounded by the variance of the price relatives (a general result of the difference between arithmetic and geometric means). We also suggest that the Wiener process would be useful for forecasting and back-casting price indices (see Case 2 in the Empirical Study). However, please note that using continuous time stochastic models for real (discrete time) price cases has some limitations: (a) we must have long enough time series of high-frequency data to estimate the parameters of the used model; (b) we should verify the assumptions of the model and we have to make sure that the used model fits to the analysed data set; (c) we have to select a group of products from the CPI basket that behave according to the nature of the model. For instance, the GBM price model is not appropriate for the case of weakly seasonal goods, since the price of these goods has a rather periodical form. Thus, the assumption of the GBM model cannot be true for all the items included in CPI baskets. However, as it was shown based on examples provided, the presented methods work quite effectively in the case of prices showing a trend over time.

Please also note that there are many other interesting continuous-time stochastic models in the literature that could be applied in price modelling at the elementary level. For example, we can encounter interesting generalisations of the GBM model (Kühn and Neu 2008; You-Sheng and Cheng-Hsun 2011), a multivariate version of this model (Hu 2000), 
the GBM model with jumps and other extensions (Kou 2002; Hong-Bae and Tae-Jun 2015), or a more general process than the one considered in which drifts and volatilities are changeable over time. In fact, these models are much more complicated and the estimation of their parameters is not easy. In our opinion, they are more likely to be used in the nearest future, when scanner data (high frequency data) become fully available, and we know the real nature of price volatilities. The application of such extended continuous-time stochastic models for price index modelling is part of our potential future work.

\section{References}

Balk, B.M. 2005. "Price Indexes for Elementary Aggregates: The Sampling Approach." Journal of Official Statistics 21(4): 675-699. Available at: https://www.scb.se/contentassets/ca21 efb41fee $47 \mathrm{~d} 293$ bbee $5 \mathrm{bf} 7 \mathrm{be} 7 \mathrm{fb} 3 /$ price-indexes-for-elementary-aggregatesthe-sampling-approach.pdf (accessed September 2020).

Barlow, M.T. 2002. “A Diffusion Model for Electricity Prices.” Mathematical Finance 12 (4): 287-298. DOI: https://doi.org/10.1111/j.1467-9965.2002.tb00125.x.

Białek, J. 2013. "Measuring Average Rate of Return of Pensions: A Discrete, Stochastic and Continuous Price Index Approaches." International Journal of Statistics and Probability 2(4): 56-63. DOI: https://doi.org/10.5539/ijsp.v2n4p56.

Białek, J. 2015. "Generalization of the Divisia price and quantity indices in a stochastic model with continuous time." Communications in Statistics: Theory and Methods 44(2): 309-328. DOI: https://doi.org/10.1080/03610926.2014.968738.

Boskin, M.S. 1996. (Chair) Advisory Commission to Study the Consumer Price Index. "Towards a More Accurate Measure of the Cost of Living." Final report for the Senate Finance Committee. Washington D.C., Available at: https://www.ssa.gov/history/ reports/boskinrpt.html.

Boskin, M.S., E.R. Dulberger, R.J. Gordon, Z. Griliches, and D.W. Jorgenson. 1998. "Consumer prices in the consumer price index and the cost of living." Journal of Economic Perspectives 12(1): 3-26. DOI: https://doi.org/10.1257/jep. 12.1.3.

Bureau of Labor Statistics. 2001. The experimental CPI using geometric means (CPI-U$\mathrm{XG})$.

Carruthers, A.G., D.J. Sellwood, and P.W. Ward. 1980. "Recent developments in the retail price index." The Statistician 29(1): 1-32. DOI: https://doi.org/10.2307/2987492.

Carli, G. 1804. Del valore e della proporzione de'metalli monetati. In: Scrittori Classici Italiani di Economia Politica 13: 297-336. Available at: https://books.google.it/ books?id=v31JAAAAMAAJ.

Cobb, B.R., R. Rumi, and A. Salmeron. 2012. “Approximation the Distribution of a Sum of Log-normal Random Variables". Paper presented at the Sixth European Workshop on Probabilistic Graphical Models, Granada, Spain, 2012. Available at: http://leo.ugr. es/pgm2012/proceedings/eproceedings/cobb_approximating.pdf.

Consumer Price Index Manual. Theory and practice. 2004. ILO/IMF/OECD/UNECE/Eurostat/The World Bank, International Labour Office (ILO), Geneva. Available at: https:/www.ilo.org/wcmsp5/groups/public/--dgreports/--stat/documents/presentation/wcms_331153.pdf. 
Dalén, J. 1992. "Computing Elementary Aggregates in the Swedish Consumer Price Index.” Journal of Official Statistics 8(2): 129-147. Available at: https://www.scb.se/contentassets/ca21 efb41fee47d293bbee 5bf7be7fb3/computing-elementary-aggregatesin-the-swedish-consumer-price-index.pdf (accessed September 2020).

Dalén, J. 1994. "Sensitivity Analyses for Harmonising European Consumer Price Indices": 147-171 in International Conference on Price Indices: Papers and Final Report, First Meeting of the International Working Group on Price Indices, November 1994, Ottawa, Statistics Canada. Available at: https:/www.ottawagroup.org/Ottawa/ ottawagroup.nsf/home/Meeting+1/\$file/1994+1st+Meeting+-+Dal\%C3\%A9n $+\mathrm{J} \% \mathrm{C} 3 \%$ B6rgen +-+ Sensitivity + Analyses + for + Harmonising + European + Consumer+Price+Indices.pdf.

Dalén, J. 1999. "A Note on the Variance of the Sample Geometric Mean. Research Report 1." Department of Statistics, Stockholm University, Stockholm.

Diewert, W.E. 1995. "Axiomatic and economic approaches to elementary price indexes." Discussion Paper No. 95-01. Department of Economics, University of British Columbia, Vancouver, Canada. Available at: https://economics.ubc.ca/files/2013/06/pdf_paper_erwin-diewert-95-01-axiomatic-economic-approaches.pdf.

Diewert, W.E. 2012. "Consumer price statistics in the UK." Office for National Statistics, Newport. Available at: https://www.unece.org/fileadmin/DAM/stats/documents/ece/ ces/ge.22/2014/WS1/WS1_1_Diewert_on_Diewert_Consumer_Price_Statistics_in_ the_UK_v.7_06.08__Final.pdf.

Dorfman, A.H., S. Leaver, and J. Lent. 1999. "Some observations on price index estimators." U.S. Bureau of Labor Statistics (BLS) Statistical Policy Working Paper 29(2). Washington D.C. Available at: https://www.bls.gov/osmr/research-papers/1999/ st990080.htm.

Eichhorn, W., and J. Voeller. 1976. "Theory of the Price Index." Lecture Notes in Economics and Mathematical Systems 140. Berlin-Heidelberg-New York: SpringerVerlag. DOI: https://doi.org/10.1007/978-3-642-45492-9.

Evans, B. 2012. International comparison of the formula effect between the CPI and RPI. Office for National Statistics. Newport. Available at: https://pdfs.semanticscholar.org/ 6667/3228f9665c9cd31011da1ef932eee394afa9.pdf?_ga $=2.36133664 .1832035$ 111.1583498850-1342646647.1583498850. Published online in 2012.

Fenton, L.F. 1960. "The sum of log-normal probability distributions in scatter transmission systems." IRE Transactions on Communications Systems 8(1): 57-67. DOI: https://doi.org/10.1109/tcom.1960.1097606.

Gajek, L., and M. Kałuszka. 2004. "On the average rate of return in a continuous time stochastic model.” Working paper. Technical University of Lodz, Poland. Available at: https://www.researchgate.net/publication/270892015_ON_THE_AVERAGE_RATE_OF_RETURN_IN_A_CONTINUOUS_TIME_STOCHASTIC_MODEL.

Greenlees, J.S. 2001. "Random errors and superlative indexes." Working Paper 343. Bureau of Labour Statistics, Washington D.C. Available at: https://www.bls.gov/pir/journal/gj09.pdf.

Hardy, G.H., J.E. Littlewood, and G. Polya. 1934. Inequalities. Cambridge: Cambridge University Press. DOI: https://doi.org/10.2307/3605504. 
Hong-Bae, K., and P. Tae-Jun. 2015. "The Behavior Comparison between Mean Reversion and Jump Diffusion of CDS Spread." Eurasian Journal of Economics and Finance 3(4): 8-21. DOI: https://doi.org/10.15604/ejef.2015.03.04.002.

$\mathrm{Hu}$, Y. 2000. "Multi-dimensional geometric Brownian motions, Onsager-Machlup functions, and applications to mathematical finance." Acta Mathematica Scientia 20(3): 341-358. DOI: https://doi.org/10.1016/s0252-9602(17)30641-0.

Hull, J. 2018. Options, Futures, and other Derivatives (10 ed.). Boston: Pearson.

Jakubowski, J., A. Palczewski, M. Rutkowski, and L. Stettner. 2003. Matematyka finansowa. Instrumenty pochodne. Warszawa: Wydawnictwa Naukowo-Techniczne.

Jevons, W.S. 1865. "On the variation of prices and the value of the currency since 1782." J. Statist. Soc. Lond. 28: 294-320. DOI: https://doi.org/10.2307/2338419.

Kou, S.G. 2002. "A jump-diffusion model for option pricing." Management Science 48: 1086-1101. DOI: https://doi.org/10.1287/mnsc.48.8.1086.166.

Kühn, R., and P. Neu. 2008. "Intermittency in an interacting generalization of the geometric Brownian motion model." Journal of Physics A: Mathematical and Theoretical 41 (2008): 1-12. DOI: https://doi.org/10.1088/1751-8113/41/32/324015.

Levell, p. 2015. "Is the Carli index flawed?: assessing the case for the new retail price index RPIJ.” J. R. Statist. Soc. A 178(2): 303-336. DOI: https://doi.org/10.1111/rssa. 12061.

McClelland, R., and M. Reinsdorf. 1999. "Small Sample Bias in Geometric Mean and Seasoned CPI Component Indexes.” Bureau of Labor Statistics Working Paper No. 324, Washington D.C. Available at: https://stats.bls.gov/osmr/research-papers/1999/pdf/ec990050.pdf.

Meade, N. 2010. "Oil prices - Brownian motion or mean reversion? A study using a one year ahead density forecast criterion.” Energy Economics 32 (2010): 1485-1498. DOI: https://doi.org/10.1016/j.eneco.2010.07.010.

Moulton, B.R., and K.E. Smedley. 1995. "A comparison of estimators for elementary aggregates of the CPI." Paper present at Second Meeting of the International Working Group on Price Indices. Stockholm, Sweden. Available at: https://www.ottawagroup.org/Ottawa/ottawagroup.nsf/4a256353001af3ed4b2562bb00121564/8e98d9c3d6e9363eca25727500004401/\$FILE/1995\%202nd\%20Meeting\%20-\%20A\%20comparison\%20of\%20esti.

Nwafor, C.N., and A.A. Oyedele. 2017. "Simulation and Hedging Oil Price with Geometric Brownian Motion and Single-Step Binomial Price Model." European Journal of Business and Management 9(9): 68-81. Available at: https://researchonline.gcu.ac.uk/files/24776117/Simulation_of_Crude_Oil_Prices_EJBM_Vol.9_No.pdf.

Office for National Statistics. 2013. National Statistician announces outcome of consultation on RPI. Office for National Statistics, Newport. Available at: https:// webarchive.nationalarchives.gov.uk/20160111163943/http:/www.ons.gov.uk/ons/dcp29904_295002.pdf.

Oksendal, B. 2003. Stochastic Differential Equations: An Introduction with Applications. Berlin: Springer. DOI: https://doi.org/10.1007/978-3-642-14394-6_5.

Privault, N. 2012. "An Elementary Introduction to Stochastic Interest Rate Modeling." Advanced Series on Statistical Science \& Applied Probability: Volume 16, Word Scientific. DOI: https://doi.org/10.1142/8416. 
Ross, S.M. 2014. "Variations on Brownian Motion." In Introduction to Probability Models (11th ed.), 612-14. Amsterdam: Elsevier.

Schultz, B. 1995. "Choice of price index formulae at the micro-aggregation level: The Canadian Empirical evidence." (Version updated in June 1995) Paper presented at the first meeting of the Ottawa Group on Price indices, Canada. Available at: https://www. ottawagroup.org/Ottawa/ottawagroup.nsf/home/Meeting+1/\$file/1994\%201st\%20Meeting $\% 20-\% 20$ Schultz $\% 20$ Bohdan $\% 20-\% 20$ Choice $\% 20$ of $\% 20$ Price $\% 20$ Index $\%$ 20Formulae\%20at\%20the\%20Micro-Aggregation\%20Level\%20The\%20Canadian\%20Empirical\%20Evidence\%202nd\%20Edition.pdf.

Silver, H., and S. Heravi. 2007. "Why elementary price index number formulas differ: Evidence on price dispersion." Journal of Econometrics, 140 (2007): 874-883. DOI: https://doi.org/10.1016/j.jeconom.2006.07.017.

UK Statistics Authority. 2013. Consultation on the Retail Prices Index. Statement. UK Statistics Authority, London. Available at: http://www.statisticauthority.gov.uk/news/ statement-consultation-on-the-retail-prices-index-10012013.pdf.

Von der Lippe, p. 2007. Index Theory and Price Statistics. Peter Lang Verlag. DOI: https://doi.org/10.3726/978-3-653-01120-3.

White, A.G. 1999. "Measurement Biases in Consumer Price Indexes." International Statistical Review, 67(3): 301-325. DOI: https://doi.org/10.1111/j.1751-5823.1999. tb00451.

Yu-Sheng H., and W. Cheng-Hsun 2011. "A Generalization of Geometric Brownian Motion with Applications." Communications in Statistics - Theory and Methods, 40(12): 2081-2103. DOI: https://doi.org/10.1080/03610921003764167.

Received November 2020

Revised May 2019

Accepted March 2020 